\title{
Recent Progress in the Chemistry of Synthetic Dyes
}

\author{
by Prof. Dr. K. Venkataraman \\ Director of National Chemical Laboratory \\ Poona, India
}

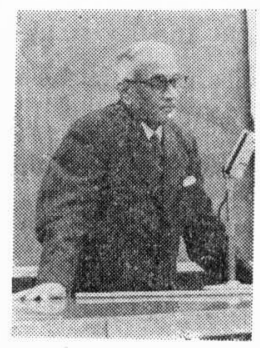

The chemistry of synthetic dyes is becoming out of fashion in universities and it will be the purpose of this talk to show that it continues to offer an exciting and rewarding field for exploration.

Examples will be cited of reactions discovered originally in connection with synthetic dyes and now finding wider applications. The benzidine transformation, discovered about a hundredyears ago, is still a favourite topic for physical organic chemists and the mechanism has not yet been fully elucidated. Bucherer published in 1904 the first systematic study of the reaction named after him, but it was only three years ago that Reiche and Seeboth demonstrated its real mechanism. A reaction reported by Ettling in 1845 has led in the hands of Toland and Kaeding to a new process for converting toluene through benzoic acid to phenol or salicylic acid. Diazotization was discovered by Griess in 1858, and just about 100 years later Tedder described a method for the direct introduction of the diazonium group into aromatic compounds by the action of nitrous acid, the yields being adequate for preparative purposes when they contain activating groups. Hyman Laboratories (1954) have patented a very interesting series of reactions which are useful for the preparation of relatively inaccessible naphthalene derivatives such as 2-nitronaphthalene, and 2-bromo-3-nitronaphthalene, and which also provide a new route to 2-hydroxy-3naphthoic acid; naphthalene acts as a dienophile in the Diels-Alder reaction with hexachlorocyclopentadiene, and after nitration or halogenation of the adduct dissociation by heating regenerates hexachlorocyclopentadiene and yields $\beta$-substituted naphthalenes.

Numerous reactions, including some which are well-known and some which are buried in patent literature, remain to be investigated for their mecha- nisms and for their application to wider synthetic purposes. One example is the Marschalk reaction which has proved to be useful in the synthesis of naturally occurring anthraquinone pigments, and which will probably be useful for the synthesis of disperse dyes for polyester and other synthetic fibres. Incidentally, in the search for new disperse dyes many types of heterocyclic compounds have been synthesized.

A major development in recent years is the discovery of reactive dyes, and many hundreds of patents have been taken on derivatives of chanuric chloride, tetrachloropyrimidine, sulphonylfluorides, vinyl sulphones, and compounds containing other groups capable of entering into covalent combination with the primary hydroxyl groups of cellulose. Reactive dyes are by no means free from technica 1 disadvantages, because the reactive group obviously can combine with water as well as thecellulose hydroxyls, resulting in instability on prolonged storage and loss of colour in the dye-bath. The anthraquinonoid vat dye grades of fastness have not yet been attained, and there is no reason to believe that the reactive dyes will displace the wellestablished azoic and vat dyes. In connection with vinyl sulphones, an application in a totally different area (Kader and Stirling, 1964) is the action of a base on a $\beta$-carbamoyloxysulphone, which results in a $\beta$-elimination useful for the protection of amino groups in peptide synthesis.

In view of the increasing demand for pigments with high fastness properties for synthetic fibres, printing inks, plastics and automobile finishes, extensive work is in progress to modify textile dyes and to construct new structures. Dioxazines, fluorubines and, most notable of all, quinoacridones, are exam- 
ples. Linear trans-quinacridone was described by Liebermann in 1935, but after nearly a quarter of a century Du Pont converted the compound into three pigments of brilliant shades from red to violet, the difference being in crystal modification and particle size.

Although sulphur dyes are still largely in use, no attempt is being made to throw new light on the reactions involved and the structures of the dyes. There are tough problems of separation, purification and crystallization, which can probably be overcome to some extent by modern chromatographic techniques. In his book on Mechanism of Sulphur Reactions (1962) Pryor has tried "to provide a framework of mechanisms upon which some of the reactions of sulphur and sulphur compounds can be organized and some easily understood," but the disdain with which the academic chemist looks on synthetic dyes is reflected by the fact that on reference whatsoever is made to sulphur dyes. Incidentally, a recent development of considerable interest is the synthesis of sulphur dyes (Hyaman colours) by Hiyama (1959), involving the replacement of chlorine in cyanuric chloride by thiol grops. Remarkably clear shades on cotton, viscose and nylon, specially on vinylon, are produced. The theoretical basis of the purity of the shades is the insulation of the chromophore from thiol groups by means of the triazine ring.

Work on anthraquinonoid vat dyes and azoic coupling components carried out in Bombay and Poona may be briefly reviewed. Apart from their technical importance the anthraquinonoid vat dyes are of interest because they include complex homocyclic and heterocyclic compounds which present many problems for research on structure determination, degradation of very stable ring systems, synthetic methods, reaction mechanism, steric effects in light absorption, and photochemical activity. Electronic absorption spectra and infrared spectra have shown that Indanthrene Khaki GG has the tetracarbazole structure. The mechanism of the cyclization of anthrimides to carbazoles is being investigated. The orientation of the nitro group in nitrated dibenzanthrone (Indanthrene Black $\mathrm{BB}$ ) has been proved by synthesis of the corresponding amine. The structure originally assigned by Bally to benzanthronequinoline has been revised. The absorption spectra of dibenzanthrone and ethers derived from it have been studied and interpreted in terms of steric and resonance effects. Dibenzanthrone derivatives are attractive for studying the relation between molecular structure and semiconductor properties.

A reaction of $o$-hydroxybenzamides, useful for the hydrolysis of naphthols and identification of the acid and amine components, is their behaviour towards. cyanuric chloride and 2,4-dinitrochlorobenzene, both of which attack the amide nitrogen and not the phenolic hydroxyl group. The reaction proceeds by a direct displacement of the halogen by the amide nitrogen involving intramolecular nucleophilic catalysis by the phenoxide oxygen.

Although there are obvious experimental difficulties, work has been initiated on the NMR spectra of aromatic sulphonic acids and of the sulphuric esters. of anthrahydroquinone derivatives in the hope of obtaining data on the orientation of substituents in certain intermediates and dyes.

In the lecture hall of the Department of Chemistry, the Faculty of Science, the University of Tokyo, on April 30, 1964 